\title{
可压缩 MHD 方程的正则性与能量守恒
}

别群益 ${ }^{1,2}$, 康玲萍 ${ }^{1}$, 王其如 ${ }^{3 *}$, 姚正安 ${ }^{3}$

1. 三峡大学理学院, 宜昌 443002 ;

2. 三峡大学三峡数学研究中心, 宜昌 443002 ;

3. 中山大学数学学院, 广州 510275

E-mail: qybie@126.com,2332891948@qq.com, mcswqr@mail.sysu.edu.cn, mcsyao@mail.sysu.edu.cn

收稿日期: 2020-12-05；接受日期: 2021-05-14；网络出版日期: 2021-11-03; * 通信作者

国家自然科学基金 (批准号: 11871305, 11971496 和 12071491) 和三峡大学学位论文培优基金 (批准号: 2021SSPY147) 资助项目

摘要 本文研究可压缩理想 MHD (magnetohydrodynamics) 系统解的正则性与能量守恒问题, 给出使 得该系统保持能量守恒时其解关于正则性的两个充分条件, 其中 $(t, x) \in(0, T) \times \mathbb{T}^{d}(d \geqslant 1)$. 结论的证 明主要利用类似于 Constantin 等 (1994) 研究匀质不可压缩 Euler 方程时所采用的交换子估计.

关键词 可压缩 MHD 方程 正则性 能量守恒

MSC (2020) 主题分类 35D $30,35 \mathrm{~B} 65,35 \mathrm{Q} 35,76 \mathrm{~W} 05$

\section{1 引言}

在磁流体动力学中, MHD 方程刻画了液体金属、等离子体、盐水和电解质中导电流体的动力学 行为 (参见文献 [1-3]). 本文研究如下可压缩理想 MHD 系统的正则性与能量守恒的关系:

$$
\left\{\begin{array}{l}
\partial_{t}(\rho u)+\operatorname{div}(\rho u \otimes u)+\nabla p=\operatorname{curl} B \times B \\
\partial_{t} \rho+\operatorname{div}(\rho u)=0 \\
\partial_{t} B=\operatorname{curl}(u \times B) \\
\operatorname{div} B=0
\end{array}\right.
$$

其中, $u$ 和 $B$ 分别表示速度和磁场, $\rho$ 是密度, $p$ 是压力, 压力 $p$ 是密度 $\rho$ 满足一定条件下的给定函数. 这里所考虑的空间维数是 $d(d \geqslant 1)$ 维.

流体动力学方程弱解的能量守恒问题是近年来的一个热点问题. 对于不可压缩 Euler 方程, 自从 1949 年 Onsager ${ }^{[4]}$ 提出其著名的 Onsager 猜想后, 已经有了许多重要的研究成果. 受湍流定律的启发, Onsager 首先猜想: 存在一个阈值, 当弱解的正则性超过这个阈值时能量守恒. 具体地, 对于 3 维 Euler 
方程在 Hölder 空间 $C^{\alpha}$ 中的任意弱解, 当指标 $\alpha>\frac{1}{3}$ 时能量守恒, 而当 $\alpha<\frac{1}{3}$ 时存在能量耗散的弱 解. Eyink ${ }^{[5]}$ 在较强的假设条件下证明了解的能量守恒. 紧接着, Constantin 等 ${ }^{[6]}$ 证明了在周期区域 上当速度场 $u \in B_{3}^{\alpha, \infty}\left(\alpha>\frac{1}{3}\right)$ 时能量守恒. Duchon 和 Robert ${ }^{[7]}$ 得到了在较弱条件下的能量守恒. 2008 年, Cheskidov 等 ${ }^{[8]}$ 利用 Littlewood-Paley 分解进一步提高了之前的结果. 需要指出的是, 上述结 果均是在全空间和周期区域上 Euler 方程的能量守恒. 对于 3 维不可压缩 Euler 方程在有界区域上的 能量守恒, 可参见文献 [9-11]. 另外对于不可压缩或可压缩 Euler 方程及 Navier-Stokes 方程的能量守 恒问题可参见文献 [12-14] 等.

对于 MHD 方程的能量守恒问题最近也有了一些结果 (参见文献 [15-18]). Gao 等 [17] 考虑了不可 压缩 MHD 方程弱解的局部能量方程. Guo 和 Tan ${ }^{[18]}$ 在能量耗散方法的基础上, 从纵向和横向分别 研究了不可压缩 MHD 系统的能量方程. Wu 和 Tan ${ }^{[15]}$ 证明了在 Besov 空间的正则性条件下, 非匀 质不可压缩 MHD 系统弱解的全局能量守恒. 而对于在有界区域上的 3 维不可压缩理想 MHD 方程, Wang 和 Zuo ${ }^{[16]}$ 得到了具有内部 Besov 正则性弱解的能量守恒.

上述关于 MHD 方程的能量守恒及耗散问题, 考虑的均是不可压缩情形, 而关于可压缩情形的能 量守恒的结果还很少. 最近, 文献 [19] 利用文献 [6,13] 中的方法研究了可压缩 MHD 方程的正则性 及能量守恒问题. 本文同样利用文献 [6] 中研究不可压缩 Euler 方程时所采用的交换子估计, 以及文 献 $[13,20]$ 中的正则化方法, 得到了可压缩理想 MHD 方程能量守恒的两个充分条件. 具体地, 为了得 到第一个充分条件 (定理 1.1), 不同于匀质 Euler 方程中的项 $\partial_{t} u$ (参见文献 [6]), 系统 (1.1) 中的项 $\partial_{t}(\rho u)$ 是非线性的, 这里采用在时间和空间上同时进行正则化的方法来处理该项. 由此我们在对余项 进行交换子估计时，除了空间上的 Besov 正则性假设外，还需要加上关于时间的 Besov 正则性假设. 在第二个充分条件 (定理 1.2), 对于 $\rho 、 u$ 和 $B$ 均不需要关于时间的 Besov 正则性要求, 但需要假设 条件 $\rho, u, B \in B V \cap C$, 其中 $B V$ 和 $C$ 分别表示有界变差函数和连续函数空间. 另外, 相比于可压缩 Euler 方程, 可压缩 MHD 方程还需要考虑磁场 $B$ 的正则性对系统能量守恒的影响, 即需要处理非线 性项 $\operatorname{curl} B \times B$ 以及 $\operatorname{curl}(u \times B)$. 在定理 1.1 中, 我们利用条件 $\operatorname{div} B=0$, 将 Lorentz 力项 $\operatorname{curl} B \times B$ 改写成导数形式, 并将该导数通过分部积分转移到实验函数上, 从而去掉了文献 [19] 中关于 $\operatorname{curl} B$ 的 正则性假设条件.

在给出本文的主要结论之前, 首先给出压力势的定义如下:

$$
P(\rho) \stackrel{\text { def }}{=} \rho \int_{1}^{\rho} \frac{p(r)}{r^{2}} d r .
$$

另外, 用记号 $B V(\Omega)$ 表示有界变差函数空间. 由有界变差函数空间的拓扑性质可知, 若一个光滑函数 序列 $\left\{\omega_{n}\right\}_{n=1}^{\infty}$ 在 $B V(\Omega)$ 上收玫到 $\omega$, 则意味着 $\omega_{n}$ 在 $L^{1}(\Omega)$ 上强收玫到 $\omega$, 且其梯度 $\nabla \omega_{n}$ 在有界测 度空间 $\mathcal{M}(\Omega)$ 上弱 $*$ 收敛到 $\nabla \omega$.

本文的结论是如下两个定理:

定理 1.1 令 $(\rho, u, B)$ 是系统 (1.1) 在分布意义下的弱解, 假设

$$
u \in\left(B_{3}^{\alpha, \infty} \cap L^{\infty}\right)\left((0, T) \times \mathbb{T}^{d}\right), \quad B \in B_{3}^{\beta, \infty}\left((0, T) \times \mathbb{T}^{d}\right), \quad \rho \in\left(B_{3}^{\gamma, \infty} \cap L^{\infty}\right)\left((0, T) \times \mathbb{T}^{d}\right),
$$

且 $0 \leqslant \underline{\rho} \leqslant \rho \leqslant \bar{\rho}$ 对 $(t, x) \in(0, T) \times \mathbb{T}^{d}$ 几乎处处成立, 这里 $\rho$ 、 $\bar{\rho} 、 0<\gamma \leqslant \alpha<1$ 和 $0<\beta<1$ 是常数 并满足

$$
\min \{2 \alpha+\gamma, \alpha+2 \beta, \alpha+2 \gamma\}>1
$$


另外假设 $p \in C^{2}[\underline{\rho}, \bar{\rho}]$, 则系统 $(1.1)$ 在分布意义下满足局部能量守恒, 即

$$
\partial_{t}\left(\frac{1}{2} \rho|u|^{2}+\frac{1}{2}|B|^{2}+P(\rho)\right)+\operatorname{div}\left[\left(\frac{1}{2} \rho|u|^{2}+p(\rho)+P(\rho)\right) u\right]-\operatorname{div}((u \times B) \times B)=0,
$$

其中 $(t, x) \in(0, T) \times \mathbb{T}^{d}$.

注 1.1 注意到条件 $\alpha \geqslant \gamma$, 则 $3 \alpha \geqslant \alpha+2 \gamma>1$, 因此 $\alpha$ 需满足大于 $\frac{1}{3}$. 从而与文献 $[6,13,21]$ 等 研究不可压缩或可压缩 Euler 方程能量守恒时对速度场的正则性要求一致. 从该意义上来说, 定理 1.1 中为保证可压缩 MHD 方程能量守恒其解所需满足的正则性条件是最优的.

注 1.2 文献 [19] 中关于磁场 $B$ 的条件需满足 $\operatorname{curl} B \in B_{3}^{\beta, \infty}\left((0, T) \times \mathbb{T}^{d}\right)$, 这里去掉了这个条件.

下面给出使得系统 (1.1) 保持能量守恒另一个充分条件, 这里不需要关于时间的 Besov 正则性假 设条件.

定理 1.2 假设压力 $p$ 满足

$$
p \in C^{2}(0, \infty) \cap C[0, \infty), \quad p(0)=0 .
$$

$(\rho, u, B) \in L^{\infty}\left((0, T) \times \mathbb{T}^{d}\right)$ 是系统 $(1.1)$ 在分布意义下的一个解, 且对几乎处处 $t \in[0, T]$ 满足

$$
\rho(t), u(t), B(t) \in B V \cap C\left(\mathbb{T}^{d}\right)
$$

及

$$
\rho, u, B \in L^{\infty}\left(0, T ; C\left(\mathbb{T}^{d}\right)\right), \quad \nabla \rho, \nabla u, \operatorname{curl} B \in L_{\mathrm{weak}^{*}}^{\infty}\left(0, T ; \mathcal{M}\left(\mathbb{T}^{d}\right)\right) .
$$

则系统 (1.1) 在分布意义下满足如下局部能量方程:

$$
\partial_{t}\left(\frac{1}{2} \rho|u|^{2}+\frac{1}{2}|B|^{2}+P(\rho)\right)+\operatorname{div}\left[\left(\frac{1}{2} \rho|u|^{2}+p(\rho)+P(\rho)\right) u\right]-\operatorname{div}[(u \times B) \times B]=0,
$$

其中 $(t, x) \in(0, T) \times \mathbb{T}^{d}$.

注 1.3 定理 1.1 和 1.2 的证明方法同样适用于可压缩理想 Hall-MHD 方程, 并可得到该方程相 应的局部能量守恒充分条件.

\section{2 预备知识}

\subsection{Besov 空间}

假设 $\Omega=(0, T) \times \mathbb{T}^{d}$ 或者 $\Omega=\mathbb{T}^{d}$. Besov 空间 $B_{p}^{\alpha, \infty}(\Omega)$ 由具有如下范数的函数 $f$ 所组成:

$$
\|f\|_{B_{p}^{\alpha, \infty}(\Omega)} \stackrel{\text { def }}{=}\|f\|_{L^{p}(\Omega)}+\sup _{y \in \Omega} \frac{\|f(\cdot+y)-f\|_{L^{p}(\Omega \cap(\Omega-y))}}{|y|^{\alpha}}<\infty,
$$

这里 $\Omega-y=\{x-y: x \in \Omega\}$.

\section{2 磨光化}

设 $\eta \in C_{c}^{\infty}\left(R^{N}\right)$ 是一个标准的磨光核, 这里 $N=1+d$ 或者 $N=d$. 令

$$
\eta^{\epsilon}(x)=\frac{1}{\epsilon^{N}} \eta\left(\frac{x}{\epsilon}\right) .
$$


对任意函数 $f$, 规定记号 $f^{\epsilon}=\eta^{\epsilon} * f$, 这里 $f^{\epsilon}$ 在 $\Omega^{\epsilon}=\{x \in \Omega: \operatorname{dist}(x, \partial \Omega)>\epsilon\}$ 是有意义的.

由 Besov 空间的定义, 可得到如下结论:

$$
\left\|f^{\epsilon}-f\right\|_{L^{p}(\Omega)} \leqslant C \epsilon^{\alpha}\|f\|_{B_{p}^{\alpha, \infty}(\Omega)}
$$

和

$$
\left\|\nabla f^{\epsilon}\right\|_{L^{p}(\Omega)} \leqslant C \epsilon^{\alpha-1}\|f\|_{B_{p}^{\alpha, \infty}(\Omega)} .
$$

其证明可参见文献 $[15]$.

\section{3 主要结论的证明}

\section{1 定理 1.1 的证明}

类似于文献 [13] 的方法, 磨光动量方程得

$$
\partial_{t}(\rho u)^{\epsilon}+\operatorname{div}(\rho u \otimes u)^{\epsilon}+\nabla p(\rho)^{\epsilon}=(\operatorname{curl} B \times B)^{\epsilon} .
$$

设 $\varphi \in C_{0}^{\infty}\left((0, T) \times \mathbb{T}^{d}\right)$ 是实验函数, 用 $\varphi u^{\epsilon}$ 乘以上述磨光后的方程, 然后关于时间和空间积分得

$$
\begin{aligned}
& \int_{0}^{T} \int_{\mathbb{T}^{d}} \partial_{t}(\rho u)^{\epsilon} \cdot \varphi u^{\epsilon} d x d t+\int_{0}^{T} \int_{\mathbb{T}^{d}} \operatorname{div}(\rho u \otimes u)^{\epsilon} \cdot \varphi u^{\epsilon} d x d t \\
& -\int_{0}^{T} \int_{\mathbb{T}^{d}}(\operatorname{curl} B \times B)^{\epsilon} \cdot \varphi u^{\epsilon} d x d t+\int_{0}^{T} \int_{\mathbb{T}^{d}} \varphi u^{\epsilon} \cdot \nabla p(\rho)^{\epsilon} d x d t \\
& \quad=0
\end{aligned}
$$

取 $\epsilon$ 足够小, 使得 $\operatorname{supp} \varphi \subset(\epsilon, T-\epsilon) \times \mathbb{T}^{d}$. 通过适当的交换子可将 (3.1) 改写为

$$
\begin{aligned}
\int_{0}^{T} & \int_{\mathbb{T}^{d}} \partial_{t}\left(\rho^{\epsilon} u^{\epsilon}\right) \cdot \varphi u^{\epsilon} d x d t+\int_{0}^{T} \int_{\mathbb{T}^{d}} \operatorname{div}\left((\rho u)^{\epsilon} \otimes u^{\epsilon}\right) \cdot \varphi u^{\epsilon} d x d t \\
- & \int_{0}^{T} \int_{\mathbb{T}^{d}}\left(\operatorname{curl} B^{\epsilon} \times B^{\epsilon}\right) \cdot \varphi u^{\epsilon} d x d t+\int_{0}^{T} \int_{\mathbb{T}^{d}} \varphi u^{\epsilon} \cdot \nabla p\left(\rho^{\epsilon}\right) d x d t \\
& \stackrel{\text { def }}{=} R_{1}^{\epsilon}+R_{2}^{\epsilon}+R_{3}^{\epsilon}+R_{4}^{\epsilon},
\end{aligned}
$$

其中,

$$
\begin{aligned}
& R_{1}^{\epsilon} \stackrel{\text { def }}{=} \int_{0}^{T} \int_{\mathbb{T}^{d}} \partial_{t}\left(\rho^{\epsilon} u^{\epsilon}-(\rho u)^{\epsilon}\right) \cdot \varphi u^{\epsilon} d x d t, \\
& R_{2}^{\epsilon} \stackrel{\text { def }}{=} \int_{0}^{T} \int_{\mathbb{T}^{d}} \operatorname{div}\left[(\rho u)^{\epsilon} \otimes u^{\epsilon}-(\rho u \otimes u)^{\epsilon}\right] \cdot \varphi u^{\epsilon} d x d t, \\
& R_{3}^{\epsilon} \stackrel{\text { def }}{=} \int_{0}^{T} \int_{\mathbb{T}^{d}}\left[(\operatorname{curl} B \times B)^{\epsilon}-\left(\operatorname{curl} B^{\epsilon} \times B^{\epsilon}\right)\right] \cdot \varphi u^{\epsilon} d x d t, \\
& R_{4}^{\epsilon} \stackrel{\text { def }}{=} \int_{0}^{T} \int_{\mathbb{T}^{d}} \nabla\left(p\left(\rho^{\epsilon}\right)-p(\rho)^{\epsilon}\right) \cdot \varphi u^{\epsilon} d x d t .
\end{aligned}
$$


下面依次计算 (3.2) 中的各项. 对于左边的第 1 项, 有

$$
\int_{0}^{T} \int_{\mathbb{T}^{d}} \partial_{t}\left(\rho^{\epsilon} u^{\epsilon}\right) \cdot \varphi u^{\epsilon} d x d t=\int_{0}^{T} \int_{\mathbb{T}^{d}}\left(\varphi \partial_{t} \rho^{\epsilon}\left|u^{\epsilon}\right|^{2}+\frac{1}{2} \varphi \rho^{\epsilon} \partial_{t}\left|u^{\epsilon}\right|^{2}\right) d x d t .
$$

对于 (3.2) 左边的第 2 项, 首先磨光连续性方程 $(1.1)_{2}$, 有

$$
\partial_{t} \rho^{\epsilon}+\operatorname{div}(\rho u)^{\epsilon}=0
$$

直接计算, 可得

$$
\begin{aligned}
\int_{0}^{T} & \int_{\mathbb{T}^{d}} \operatorname{div}\left((\rho u)^{\epsilon} \otimes u^{\epsilon}\right) \cdot \varphi u^{\epsilon} d x d t \\
& =-\int_{0}^{T} \int_{\mathbb{T}^{d}}\left((\rho u)^{\epsilon} \otimes u^{\epsilon}\right): \nabla\left(\varphi u^{\epsilon}\right) d x d t \\
& =-\int_{0}^{T} \int_{\mathbb{T}^{d}} \varphi\left((\rho u)^{\epsilon} \otimes u^{\epsilon}\right): \nabla u^{\epsilon} d x d t-\int_{0}^{T} \int_{\mathbb{T}^{d}}\left((\rho u)^{\epsilon} \otimes u^{\epsilon}\right):\left(\nabla \varphi \otimes u^{\epsilon}\right) d x d t \\
& =-\frac{1}{2} \int_{0}^{T} \int_{\mathbb{T}^{d}} \varphi(\rho u)^{\epsilon} \cdot \nabla\left|u^{\epsilon}\right|^{2} d x d t-\int_{0}^{T} \int_{\mathbb{T}^{d}}\left((\rho u)^{\epsilon} \cdot \nabla \varphi\right)\left|u^{\epsilon}\right|^{2} d x d t \\
& =\frac{1}{2} \int_{0}^{T} \int_{\mathbb{T}^{d}} \operatorname{div}\left(\varphi(\rho u)^{\epsilon}\right)\left|u^{\epsilon}\right|^{2} d x d t-\int_{0}^{T} \int_{\mathbb{T}^{d}}\left((\rho u)^{\epsilon} \cdot \nabla \varphi\right)\left|u^{\epsilon}\right|^{2} d x d t \\
& =\frac{1}{2} \int_{0}^{T} \int_{\mathbb{T}^{d}}\left(\nabla \varphi \cdot(\rho u)^{\epsilon}+\varphi \operatorname{div}(\rho u)^{\epsilon}\right)\left|u^{\epsilon}\right|^{2} d x d t-\int_{0}^{T} \int_{\mathbb{T}^{d}}\left((\rho u)^{\epsilon} \cdot \nabla \varphi\right)\left|u^{\epsilon}\right|^{2} d x d t \\
& =-\frac{1}{2} \int_{0}^{T} \int_{\mathbb{T}^{d}} \varphi \partial_{t} \rho^{\epsilon}\left|u^{\epsilon}\right|^{2} d x d t-\frac{1}{2} \int_{0}^{T} \int_{\mathbb{T}^{d}}\left((\rho u)^{\epsilon} \cdot \nabla \varphi\right)\left|u^{\epsilon}\right|^{2} d x d t .
\end{aligned}
$$

为了计算 $(3.2)$ 左边的第 3 项, 我们磨光 $(1.1)_{3}$ 并乘以 $B^{\epsilon}$, 得

$$
\frac{1}{2} \partial_{t}\left|B^{\epsilon}\right|^{2}=\operatorname{curl}(u \times B)^{\epsilon} \cdot B^{\epsilon} .
$$

上式结合公式 $\operatorname{div}(a \times b)=\operatorname{curl} a \cdot b-a \cdot \operatorname{curl} b$, 有

$$
\begin{aligned}
\int_{0}^{T} & \int_{\mathbb{T}^{d}}\left(\operatorname{curl} B^{\epsilon} \times B^{\epsilon}\right) \cdot \varphi u^{\epsilon} d x d t \\
= & -\int_{0}^{T} \int_{\mathbb{T}^{d}} \varphi\left(u^{\epsilon} \times B^{\epsilon}\right) \cdot \operatorname{curl} B^{\epsilon} d x d t \\
= & \int_{0}^{T} \int_{\mathbb{T}^{d}} \varphi \operatorname{div}\left(\left(u^{\epsilon} \times B^{\epsilon}\right) \times B^{\epsilon}\right) d x d t-\int_{0}^{T} \int_{\mathbb{T}^{d}} \varphi \operatorname{curl}\left(u^{\epsilon} \times B^{\epsilon}\right) \cdot B^{\epsilon} d x d t \\
= & \int_{0}^{T} \int_{\mathbb{T}^{d}} \varphi \operatorname{div}\left(\left(u^{\epsilon} \times B^{\epsilon}\right) \times B^{\epsilon}\right) d x d t-\int_{0}^{T} \int_{\mathbb{T}^{d}} \varphi \operatorname{curl}(u \times B)^{\epsilon} \cdot B^{\epsilon} d x d t \\
& -\int_{0}^{T} \int_{\mathbb{T}^{d}} \varphi \operatorname{curl}\left[\left(u^{\epsilon} \times B^{\epsilon}\right)-(u \times B)^{\epsilon}\right] \cdot B^{\epsilon} d x d t \\
& \stackrel{\text { def }}{=}-\frac{1}{2} \int_{0}^{T} \int_{\mathbb{T}^{d}} \varphi \partial_{t}\left|B^{\epsilon}\right|^{2} d x d t-\int_{0}^{T} \int_{\mathbb{T}^{d}}\left(u^{\epsilon} \times B^{\epsilon}\right) \times B^{\epsilon} \cdot \nabla \varphi d x d t+R_{5}^{\epsilon} \\
= & \frac{1}{2} \int_{0}^{T} \int_{\mathbb{T}^{d}} \partial_{t} \varphi\left|B^{\epsilon}\right|^{2} d x d t-\int_{0}^{T} \int_{\mathbb{T}^{d}}\left(u^{\epsilon} \times B^{\epsilon}\right) \times B^{\epsilon} \cdot \nabla \varphi d x d t+R_{5}^{\epsilon} .
\end{aligned}
$$


对于 (3.2) 左边第 4 项, 利用磨光的质量方程 (3.4) 及压力势 $P$ 的定义, 有

$$
P^{\prime}\left(\rho^{\epsilon}\right)\left(\partial_{t} \rho^{\epsilon}+u^{\epsilon} \cdot \nabla \rho^{\epsilon}+\rho^{\epsilon} \operatorname{div} u^{\epsilon}\right)=P^{\prime}\left(\rho^{\epsilon}\right) \operatorname{div}\left(\rho^{\epsilon} u^{\epsilon}-(\rho u)^{\epsilon}\right) .
$$

再由链式法则, 上式变为

$$
\partial_{t} P\left(\rho^{\epsilon}\right)+u^{\epsilon} \cdot \nabla P\left(\rho^{\epsilon}\right)+P^{\prime}\left(\rho^{\epsilon}\right) \rho^{\epsilon} \operatorname{div} u^{\epsilon}=P^{\prime}\left(\rho^{\epsilon}\right) \operatorname{div}\left(\rho^{\epsilon} u^{\epsilon}-(\rho u)^{\epsilon}\right) .
$$

另外, 由 $P$ 的定义, 有

$$
\rho^{\epsilon} P^{\prime}\left(\rho^{\epsilon}\right)=P\left(\rho^{\epsilon}\right)+p\left(\rho^{\epsilon}\right) .
$$

由此可计算 (3.2) 左边第 4 项如下:

$$
\begin{aligned}
\int_{0}^{T} & \int_{\mathbb{T}^{d}} \varphi u^{\epsilon} \cdot \nabla p\left(\rho^{\epsilon}\right) d x d t \\
= & -\int_{0}^{T} \int_{\mathbb{T}^{d}} \nabla \varphi \cdot u^{\epsilon} p\left(\rho^{\epsilon}\right) d x d t-\int_{0}^{T} \int_{\mathbb{T}^{d}} \varphi p\left(\rho^{\epsilon}\right) \operatorname{div} u^{\epsilon} d x d t \\
= & -\int_{0}^{T} \int_{\mathbb{T}^{d}} \nabla \varphi \cdot u^{\epsilon} p\left(\rho^{\epsilon}\right) d x d t-\int_{0}^{T} \int_{\mathbb{T}^{d}} \varphi\left[\rho^{\epsilon} P^{\prime}\left(\rho^{\epsilon}\right)-P\left(\rho^{\epsilon}\right)\right] \operatorname{div} u^{\epsilon} d x d t \\
= & -\int_{0}^{T} \int_{\mathbb{T}^{d}} \nabla \varphi \cdot u^{\epsilon} p\left(\rho^{\epsilon}\right) d x d t+\int_{0}^{T} \int_{\mathbb{T}^{d}} \varphi\left[\partial_{t} P\left(\rho^{\epsilon}\right)+u^{\epsilon} \cdot \nabla P\left(\rho^{\epsilon}\right)+P\left(\rho^{\epsilon}\right) \operatorname{div} u^{\epsilon}\right] d x d t \\
& -\int_{0}^{T} \int_{\mathbb{T}^{d}} \varphi P^{\prime}\left(\rho^{\epsilon}\right) \operatorname{div}\left(\rho^{\epsilon} u^{\epsilon}-(\rho u)^{\epsilon}\right) d x d t \\
= & -\int_{0}^{T} \int_{\mathbb{T}^{d}} \nabla \varphi \cdot u^{\epsilon} p\left(\rho^{\epsilon}\right) d x d t-\int_{0}^{T} \int_{\mathbb{T}^{d}} \partial_{t} \varphi P\left(\rho^{\epsilon}\right) d x d t-\int_{0}^{T} \int_{\mathbb{T}^{d}} \nabla \varphi \cdot P\left(\rho^{\epsilon}\right) u^{\epsilon} d x d t \\
& -\int_{0}^{T} \int_{\mathbb{T}^{d}} \varphi P^{\prime}\left(\rho^{\epsilon}\right) \operatorname{div}\left(\rho^{\epsilon} u^{\epsilon}-(\rho u)^{\epsilon}\right) d x d t .
\end{aligned}
$$

联立 (3.2)、(3.3)、(3.5)、(3.7) 和 (3.9), 得

$$
\begin{aligned}
& \int_{0}^{T} \int_{\mathbb{T}^{d}} \partial_{t} \varphi\left(\frac{1}{2} \rho^{\epsilon}\left|u^{\epsilon}\right|^{2}+\frac{1}{2}\left|B^{\epsilon}\right|^{2}+P\left(\rho^{\epsilon}\right)\right) d x d t \\
& \quad+\int_{0}^{T} \int_{\mathbb{T}^{d}} \nabla \varphi \cdot\left(\frac{1}{2}(\rho u)^{\epsilon}\left|u^{\epsilon}\right|^{2}+p\left(\rho^{\epsilon}\right) u^{\epsilon}+P\left(\rho^{\epsilon}\right) u^{\epsilon}-\left(u^{\epsilon} \times B^{\epsilon}\right) \times B^{\epsilon}\right) d x d t \\
& \quad=:-R_{1}^{\epsilon}-R_{2}^{\epsilon}-R_{3}^{\epsilon}-R_{4}^{\epsilon}-\int_{0}^{T} \int_{\mathbb{T}^{d}} \varphi P^{\prime}\left(\rho^{\epsilon}\right) \operatorname{div}\left(\rho^{\epsilon} u^{\epsilon}-(\rho u)^{\epsilon}\right) d x d t .
\end{aligned}
$$

为了证明定理 1.1, 我们需证明当 $\epsilon \rightarrow 0$ 时, $R_{i}^{\epsilon}(i=1,2,3,4)$ 和 $\int_{0}^{T} \int_{\mathbb{T}^{d}} \varphi P^{\prime}\left(\rho^{\epsilon}\right) \operatorname{div}\left(\rho^{\epsilon} u^{\epsilon}-(\rho u)^{\epsilon}\right) d x d t$ 趋 于 0 . 事实上, 当 $\epsilon \rightarrow 0$ 时, 利用磨光函数的性质, 得

$$
\begin{aligned}
\int_{0}^{T} & \int_{\mathbb{T}^{d}} \partial_{t} \varphi\left(\frac{1}{2} \rho^{\epsilon}\left|u^{\epsilon}\right|^{2}+\frac{1}{2}\left|B^{\epsilon}\right|^{2}+P\left(\rho^{\epsilon}\right)\right) d x d t \\
& +\int_{0}^{T} \int_{\mathbb{T}^{d}} \nabla \varphi \cdot\left(\frac{1}{2}(\rho u)^{\epsilon}\left|u^{\epsilon}\right|^{2}+p\left(\rho^{\epsilon}\right) u^{\epsilon}+P\left(\rho^{\epsilon}\right) u^{\epsilon}-\left(u^{\epsilon} \times B^{\epsilon}\right) \times B^{\epsilon}\right) d x d t
\end{aligned}
$$

趋近于

$$
\int_{0}^{T} \int_{\mathbb{T}^{d}} \partial_{t} \varphi\left(\frac{1}{2} \rho|u|^{2}+\frac{1}{2}|B|^{2}+P(\rho)\right) d x d t
$$




$$
+\int_{0}^{T} \int_{\mathbb{T}^{d}} \nabla \varphi \cdot\left(\frac{1}{2} \rho|u|^{2} u+p(\rho) u+P(\rho) u-(u \times B) \times B\right) d x d t .
$$

对于 $R_{1}^{\epsilon}$, 注意到

$$
\begin{aligned}
\rho^{\epsilon} u^{\epsilon}-(\rho u)^{\epsilon}= & \left(\rho^{\epsilon}-\rho\right)\left(u^{\epsilon}-u\right)-\int_{-\epsilon}^{\epsilon} \int_{\mathbb{T}^{d}} \eta^{\epsilon}(\tau, \xi)(\rho(t-\tau, x-\xi)-\rho(t, x)) \\
& \times(u(t-\tau, x-\xi)-u(t, x)) d \xi d \tau .
\end{aligned}
$$

由此, $R_{1}^{\epsilon}$ 的第 1 部分可由分部积分公式、(2.3)、(2.4) 及定理 1.1 的假设, 当 $\epsilon \rightarrow 0$ 时, 有

$$
\begin{aligned}
& \left|\int_{0}^{T} \int_{\mathbb{T}^{d}} \varphi \partial_{t}\left[\left(\rho^{\epsilon}-\rho\right)\left(u^{\epsilon}-u\right)\right] \cdot u^{\epsilon} d x d t\right| \\
& \quad \leqslant \int_{0}^{T} \int_{\mathbb{T}^{d}}\left|\partial_{t} \varphi\left(\rho^{\epsilon}-\rho\right)\left(u^{\epsilon}-u\right) \cdot u^{\epsilon}\right| d x d t+\int_{0}^{T} \int_{\mathbb{T}^{d}}\left|\varphi\left(\rho^{\epsilon}-\rho\right)\left(u^{\epsilon}-u\right) \cdot \partial_{t} u^{\epsilon}\right| d x d t \\
& \quad \leqslant C\|\varphi\|_{C^{1}} \epsilon^{\gamma} \epsilon^{\alpha}\|\rho\|_{B_{3}^{\gamma, \infty}}\|u\|_{B_{3}^{\alpha, \infty}}^{2}+C\|\varphi\|_{C^{0}} \epsilon^{\gamma} \epsilon^{\alpha} \epsilon^{\alpha-1}\|\rho\|_{B_{3}^{\gamma, \infty}\|u\|_{B_{3}^{\alpha, \infty}}^{2} \rightarrow 0 .}
\end{aligned}
$$

对于 $R_{1}^{\epsilon}$ 的第 2 部分, 根据 Fubini 定理、(2.3)、(2.4) 和分部积分公式, 可得当 $\epsilon \rightarrow 0$ 时,

$$
\begin{aligned}
\mid \int_{0}^{T} & \int_{\mathbb{T}^{d}} \varphi \partial_{t} \int_{-\epsilon}^{\epsilon} \int_{\mathbb{T}^{d}} \eta^{\epsilon}(\tau, \xi)(\rho(t-\tau, x-\xi)-\rho(t, x))(u(t-\tau, x-\xi)-u(t, x)) d \xi d \tau \cdot u^{\epsilon} d x d t \mid \\
= & \mid \int_{0}^{T} \int_{\mathbb{T}^{d}} \int_{-\epsilon}^{\epsilon} \int_{\mathbb{T}^{d}} \eta^{\epsilon}(\tau, \xi)(\rho(t-\tau, x-\xi) \\
& -\rho(t, x))(u(t-\tau, x-\xi)-u(t, x)) \cdot \partial_{t}\left(\varphi(t, x) u^{\epsilon}(t, x)\right) d \xi d \tau d x d t \mid \\
\leqslant & \int_{-\epsilon}^{\epsilon} \int_{\mathbb{T}^{d}} \int_{0}^{T} \int_{\mathbb{T}^{d}} \mid \eta^{\epsilon}(\tau, \xi)(\rho(t-\tau, x-\xi) \\
& -\rho(t, x))(u(t-\tau, x-\xi)-u(t, x)) \cdot \partial_{t} \varphi(t, x) u^{\epsilon}(t, x) \mid d x d t d \xi d \tau \\
& +\int_{-\epsilon}^{\epsilon} \int_{\mathbb{T}^{d}} \int_{0}^{T} \int_{\mathbb{T}^{d}} \mid \eta^{\epsilon}(\tau, \xi)(\rho(t-\tau, x-\xi) \\
& -\rho(t, x))(u(t-\tau, x-\xi)-u(t, x)) \cdot \varphi(t, x) \partial_{t} u^{\epsilon}(t, x) \mid d x d t d \xi d \tau .
\end{aligned}
$$

进而有

$$
\begin{aligned}
\mid \int_{0}^{T} & \int_{\mathbb{T}^{d}} \varphi \partial_{t} \int_{-\epsilon}^{\epsilon} \int_{\mathbb{T}^{d}} \eta^{\epsilon}(\tau, \xi)(\rho(t-\tau, x-\xi)-\rho(t, x))(u(t-\tau, x-\xi)-u(t, x)) d \xi d \tau \cdot u^{\epsilon} d x d t \mid \\
\leqslant & \|\varphi\|_{C^{1}} \frac{1}{\epsilon^{N}} \int_{-\epsilon}^{\epsilon} \int_{\mathbb{T}^{d}} \eta\left(\frac{\tau}{\epsilon}, \frac{\xi}{\epsilon}\right)\|\rho(t-\tau, x-\xi)-\rho(t, x)\|_{L^{3}(Q)}\|u(t-\tau, x-\xi)-u(t, x)\|_{L^{3}(Q)} \\
& \times\left\|u^{\epsilon}(t, x)\right\|_{L^{3}(Q)} d \xi d \tau \\
& +\|\varphi\|_{C^{0}} \frac{1}{\epsilon^{N}} \int_{-\epsilon}^{\epsilon} \int_{\mathbb{T}^{d}} \eta\left(\frac{\tau}{\epsilon}, \frac{\xi}{\epsilon}\right)\|\rho(t-\tau, x-\xi)-\rho(t, x)\|_{L^{3}(Q)}\|u(t-\tau, x-\xi)-u(t, x)\|_{L^{3}(Q)} \\
& \times\left\|\partial_{t} u^{\epsilon}(t, x)\right\|_{L^{3}(Q)} d \xi d \tau \\
\leqslant & \|\varphi\|_{C^{1}} \frac{1}{\epsilon^{N}} \int_{-\epsilon}^{\epsilon} \int_{\mathbb{T}^{d}} \eta\left(\frac{\tau}{\epsilon}, \frac{\xi}{\epsilon}\right) \epsilon^{\gamma}\|\rho\|_{B_{3}^{\gamma, \infty}(\Omega)} \epsilon^{\alpha}\|u\|_{B_{3}^{\alpha, \infty}(\Omega)}^{2} d \xi d \tau \\
& +\|\varphi\|_{C^{0}} \frac{1}{\epsilon^{N}} \int_{-\epsilon}^{\epsilon} \int_{\mathbb{T}^{d}} \eta\left(\frac{\tau}{\epsilon}, \frac{\xi}{\epsilon}\right) \epsilon^{\gamma}\|\rho\|_{B_{3}^{\gamma, \infty}(\Omega)} \epsilon^{\alpha}\|u\|_{B_{3}^{\alpha, \infty}(\Omega)} \epsilon^{\alpha-1}\|u\|_{B_{3}^{\alpha, \infty}(\Omega)} d \xi d \tau
\end{aligned}
$$


$\leqslant C\|\varphi\|_{C^{1}} \epsilon^{\gamma} \epsilon^{\alpha}\|\rho\|_{B_{3}^{\gamma, \infty}}\|u\|_{B_{3}^{\alpha, \infty}}^{2}+C\|\varphi\|_{C^{0}} \epsilon^{\gamma} \epsilon^{\alpha} \epsilon^{\alpha-1}\|\rho\|_{B_{3}^{\gamma, \infty}}\|u\|_{B_{3}^{\alpha, \infty}}^{2} \rightarrow 0$,

这里 $Q=\Omega \cap(\Omega+(\tau, \xi)),|(\tau, \xi)|<\epsilon$.

类似地处理 $R_{2}^{\epsilon}$. 首先有

$$
\begin{aligned}
(\rho u)^{\epsilon} \otimes u^{\epsilon}-(\rho u \otimes u)^{\epsilon}= & \left((\rho u)^{\epsilon}-\rho u\right) \otimes\left(u^{\epsilon}-u\right)-\int_{-\epsilon}^{\epsilon} \int_{\mathbb{T}^{d}} \eta^{\epsilon}(\tau, \xi)(\rho u(t-\tau, x-\xi)-\rho u(t, x)) \\
& \otimes(u(t-\tau, x-\xi)-u(t, x)) d \xi d \tau .
\end{aligned}
$$

要利用上述分解来估计 $R_{2}^{\epsilon}$, 我们要求 $\rho u \in B_{3}^{\alpha, \infty}\left((0, T) \times \mathbb{T}^{d}\right)$. 另外, 当 $\alpha \geqslant \gamma$ 时, 有 $B_{3}^{\alpha, \infty} \hookrightarrow B_{3}^{\gamma, \infty}$, 并且

$$
\begin{aligned}
\frac{\|(\rho u)(\cdot+\xi)-\rho u\|_{L^{3}}}{|\xi|^{\gamma}} & \leqslant \frac{\|\rho(u(\cdot+\xi)-u)\|_{L^{3}}}{|\xi|^{\gamma}}+\frac{\|(\rho(\cdot+\xi)-\rho) u(\cdot+\xi)\|_{L^{3}}}{|\xi|^{\gamma}} \\
& \leqslant\|\rho\|_{L^{\infty}}\|u\|_{B_{3}^{\gamma, \infty}}+\|u\|_{L^{\infty}}\|\rho\|_{B_{3}^{\gamma, \infty}} \\
& \leqslant\|\rho\|_{L^{\infty}}\|u\|_{B_{3}^{\alpha, \infty}}+\|u\|_{L^{\infty}}\|\rho\|_{B_{3}^{\gamma, \infty}} .
\end{aligned}
$$

对于 $R_{2}^{\epsilon}$ 的第 1 部分, 当 $\epsilon \rightarrow 0$ 时, 有

$$
\begin{aligned}
\mid \int_{0}^{T} & \int_{\mathbb{T}^{d}} \varphi \operatorname{div}\left[\left((\rho u)^{\epsilon}-\rho u\right) \otimes\left(u^{\epsilon}-u\right)\right] \cdot u^{\epsilon} d x d t \mid \\
\leqslant & \|\varphi\|_{C^{0}} \int_{0}^{T} \int_{\mathbb{T}^{d}}\left|\left((\rho u)^{\epsilon}-\rho u\right) \otimes\left(u^{\epsilon}-u\right): \nabla u^{\epsilon}\right| d x d t \\
& +\|\varphi\|_{C^{1}} \int_{0}^{T} \int_{\mathbb{T}^{d}}\left|\left[\left((\rho u)^{\epsilon}-\rho u\right) \otimes\left(u^{\epsilon}-u\right)\right] \cdot u^{\epsilon}\right| d x d t \\
\leqslant & C\|\varphi\|_{C^{0}} \epsilon^{\gamma} \epsilon^{\alpha} \epsilon^{\alpha-1}\|\rho u\|_{B_{3}^{\gamma, \infty}}\|u\|_{B_{3}^{\alpha, \infty}}^{2}+C\|\varphi\|_{C^{1}} \epsilon^{\gamma} \epsilon^{\alpha}\|\rho u\|_{B_{3}^{\gamma, \infty}}\|u\|_{B_{3}^{\alpha, \infty}}^{2} \\
\leqslant & C\|\varphi\|_{C^{0}} \epsilon^{\gamma+2 \alpha-1}\left(\|\rho\|_{L^{\infty}}\|u\|_{B_{3}^{\alpha, \infty}}+\|u\|_{L^{\infty}}\|\rho\|_{B_{3}^{\gamma, \infty}}\right)\|u\|_{B_{3}^{\alpha, \infty}}^{2} \\
& +C\|\varphi\|_{C^{1}} \epsilon^{\gamma+\alpha}\left(\|\rho\|_{L^{\infty}}\|u\|_{B_{3}^{\alpha, \infty}}+\|u\|_{L^{\infty}}\|\rho\|_{B_{3}^{\gamma, \infty}}\right)\|u\|_{B_{3}^{\alpha, \infty}}^{2} \rightarrow 0 .
\end{aligned}
$$

同样地, 对于 $R_{2}^{\epsilon}$ 的第 2 部分, 当 $\epsilon \rightarrow 0$ 时, 有

$$
\begin{aligned}
\mid \int_{0}^{T} & \int_{\mathbb{T}^{d}} \operatorname{div}\left[\int_{-\epsilon}^{\epsilon} \int_{\mathbb{T}^{d}} \eta^{\epsilon}(\tau, \xi)(\rho u(t-\tau, x-\xi)-\rho u(t, x))\right. \\
\otimes & (u(t-\tau, x-\xi)-u(t, x)) d \xi d \tau] \cdot \varphi u^{\epsilon} d x d t \mid \\
\leqslant & C\|\varphi\|_{C^{0}} \epsilon^{\gamma} \epsilon^{\alpha} \epsilon^{\alpha-1}\left(\|\rho\|_{L^{\infty}}\|u\|_{B_{3}^{\alpha, \infty}}+\|u\|_{L^{\infty}}\|\rho\|_{B_{3}^{\gamma, \infty}}\right)\|u\|_{B_{3}^{\alpha, \infty}}^{2} \\
& +C\|\varphi\|_{C^{1}} \epsilon^{\gamma} \epsilon^{\alpha}\left(\|\rho\|_{L^{\infty}}\|u\|_{B_{3}^{\alpha, \infty}}+\|u\|_{L^{\infty}}\|\rho\|_{B_{3}^{\gamma, \infty}}\right)\|u\|_{B_{3}^{\alpha, \infty}}^{2} \rightarrow 0 .
\end{aligned}
$$

考虑 $R_{3}^{\epsilon}$, 令

$$
\begin{aligned}
R_{3}^{\epsilon} & =\int_{0}^{T} \int_{\mathbb{T}^{d}}\left[(\operatorname{curl} B \times B)^{\epsilon}-\operatorname{curl} B^{\epsilon} \times B^{\epsilon}\right] \cdot \varphi u^{\epsilon} d x d t \\
& =\int_{0}^{T} \int_{\mathbb{T}^{d}}\left[(B \cdot \nabla B)^{\epsilon}-\frac{1}{2} \nabla\left(|B|^{2}\right)^{\epsilon}\right] \cdot \varphi u^{\epsilon} d x d t-\int_{0}^{T} \int_{\mathbb{T}^{d}}\left[\left(B^{\epsilon} \cdot \nabla B^{\epsilon}\right)-\frac{1}{2} \nabla\left(|B|^{\epsilon}\right)^{2}\right] \cdot \varphi u^{\epsilon} d x d t \\
& =\int_{0}^{T} \int_{\mathbb{T}^{d}}\left[(B \cdot \nabla B)^{\epsilon}-\left(B^{\epsilon} \cdot \nabla B^{\epsilon}\right)\right] \cdot \varphi u^{\epsilon} d x d t-\int_{0}^{T} \int_{\mathbb{T}^{d}}\left[\frac{1}{2} \nabla\left(|B|^{2}\right)^{\epsilon}-\frac{1}{2} \nabla\left(|B|^{\epsilon}\right)^{2}\right] \cdot \varphi u^{\epsilon} d x d t
\end{aligned}
$$




$$
\begin{aligned}
& =\int_{0}^{T} \int_{\mathbb{T}^{d}}\left[\partial_{i}\left(B_{i} B_{j}\right)^{\epsilon}-\partial_{i}\left(B_{i}^{\epsilon} B_{j}^{\epsilon}\right)\right] \cdot \varphi u^{\epsilon} d x d t-\int_{0}^{T} \int_{\mathbb{T}^{d}}\left[\frac{1}{2} \partial_{i}\left(\left|B_{i}\right|^{2}\right)^{\epsilon}-\frac{1}{2} \partial_{i}\left(\left|B_{i}\right|^{\epsilon}\right)^{2}\right] \cdot \varphi u^{\epsilon} d x d t \\
& \stackrel{\text { def }}{=} R_{31}^{\epsilon}+R_{32}^{\epsilon} .
\end{aligned}
$$

因为 $R_{32}^{\epsilon}$ 与 $R_{31}^{\epsilon}$ 的计算方法相同, 所以我们只对 $R_{31}^{\epsilon}$ 进行计算. 与前面相同, 先作如下分解:

$$
\begin{aligned}
B_{i}^{\epsilon} B_{j}^{\epsilon}-\left(B_{i} B_{j}\right)^{\epsilon}= & \left(B_{i}^{\epsilon}-B_{i}\right)\left(B_{j}^{\epsilon}-B_{j}\right)-\int_{-\epsilon}^{\epsilon} \int_{\mathbb{T}^{d}} \eta^{\epsilon}(\tau, \xi)\left(B_{i}(t-\tau, x-\xi)-B_{i}(t, x)\right) \\
& \times\left(B_{j}(t-\tau, x-\xi)-B_{j}(t, x)\right) d \xi d \tau .
\end{aligned}
$$

对于 $R_{31}^{\epsilon}$ 的第 1 部分, 由 Hölder 不等式, 得

$$
\begin{aligned}
& \left|\int_{0}^{T} \int_{\mathbb{T}^{d}} \partial_{i}\left[\left(B_{i}^{\epsilon}-B_{i}\right)\left(B_{j}^{\epsilon}-B_{j}\right)\right] \cdot \varphi u^{\epsilon} d x d t\right| \\
& \quad \leqslant C\|\varphi\|_{C^{0}}\left\|B_{i}^{\epsilon}-B_{i}\right\|_{L^{3}}\left\|B_{j}^{\epsilon}-B_{j}\right\|_{L^{3}}\left\|\operatorname{div} u^{\epsilon}\right\|_{L^{3}}+C\|\varphi\|_{C^{1}}\left\|B_{i}^{\epsilon}-B_{i}\right\|_{L^{3}}\left\|B_{j}^{\epsilon}-B_{j}\right\|_{L^{3}}\left\|u^{\epsilon}\right\|_{L^{3}} \\
& \quad \leqslant C\|\varphi\|_{C^{0}} \epsilon^{\beta} \epsilon^{\beta} \epsilon^{\alpha-1}\|B\|_{B_{3}^{\beta, \infty}}^{2}\|u\|_{B_{3}^{\alpha, \infty}}+C\|\varphi\|_{C^{1}} \epsilon^{\beta} \epsilon^{\beta} \epsilon^{\alpha}\|B\|_{B_{3}^{\beta, \infty}}^{2}\|u\|_{B_{3}^{\alpha, \infty}} .
\end{aligned}
$$

至于 $R_{31}^{\epsilon}$ 的第 2 部分, 利用 Fubini 定理和 Hölder 不等式, 得

$$
\begin{aligned}
& \mid \int_{0}^{T} \int_{\mathbb{T}^{d}} \partial_{i}\left(\int_{-\epsilon}^{\epsilon} \int_{\mathbb{T}^{d}} \eta^{\epsilon}(\tau, \xi)\left(B_{i}(t-\tau, x-\xi)-B_{i}(t, x)\right)\right. \\
& \left.\quad \times\left(B_{j}(t-\tau, x-\xi)-B_{j}(t, x)\right) d \xi d \tau\right) \cdot \varphi u^{\epsilon} d x d t \mid \\
& \quad \leqslant C\|\varphi\|_{C^{0}} \epsilon^{\beta} \epsilon^{\beta} \epsilon^{\alpha-1}\|B\|_{B_{3}^{\beta, \infty}}^{2}\|u\|_{B_{3}^{\alpha, \infty}}+C\|\varphi\|_{C^{1}} \epsilon^{\beta} \epsilon^{\beta} \epsilon^{\alpha}\|B\|_{B_{3}^{\beta, \infty}}^{2}\|u\|_{B_{3}^{\alpha, \infty}} .
\end{aligned}
$$

$R_{5}^{\epsilon}$ 的估计类似于 $R_{31}^{\epsilon}$ 的估计.

下面考虑 $R_{4}^{\epsilon}$. 首先注意到, 若 $p \in C^{2}([a, b])$, 则对任意 $h, h_{0} \in[a, b]$, 由中值定理得

$$
\left|p(h)-p\left(h_{0}\right)-p^{\prime}\left(h_{0}\right)\left(h-h_{0}\right)\right| \leqslant C\left(h-h_{0}\right)^{2},
$$

这里 $C$ 是不依赖于 $h$ 和 $h_{0}$ 的常数. 因此,

$$
\left|p\left(\rho^{\epsilon}(t, x)\right)-p(\rho(t, x))-p^{\prime}(\rho(t, x))\left(\rho^{\epsilon}(t, x)-\rho(t, x)\right)\right| \leqslant C\left(\rho^{\epsilon}(t, x)-\rho(t, x)\right)^{2} .
$$

类似地,

$$
\left|p(\rho(t, y))-p(\rho(t, x))-p^{\prime}(\rho(t, x))(\rho(t, y)-\rho(t, x))\right| \leqslant C(\rho(t, y)-\rho(t, x))^{2} .
$$

将 (3.14) 两边关于 $y$ 作卷积并由 Jensen 不等式, 得

$$
\left|p(\rho)^{\epsilon}(t, x)-p(\rho(t, x))-p^{\prime}(\rho(t, x))\left(\rho^{\epsilon}(t, x)-\rho(t, x)\right)\right| \leqslant C(\rho(t, \cdot)-\rho(t, x))^{2} *_{y} \eta^{\epsilon} .
$$

联立 (3.13) 和 (3.15) 得

$$
\left|p\left(\rho^{\epsilon}(t, x)\right)-p(\rho)^{\epsilon}(t, x)\right| \leqslant C\left(\rho^{\epsilon}(t, x)-\rho(t, x)\right)^{2}+C(\rho(t, \cdot)-\rho(t, x))^{2} *_{y} \eta^{\epsilon} .
$$

至此, 与前面类似, 当 $\epsilon \rightarrow 0$ 时, 我们可估计 $R_{4}^{\epsilon}$ 如下:

$$
\left|R_{4}^{\epsilon}\right|=\left|\int_{0}^{T} \int_{\mathbb{T}^{d}} \nabla\left(p\left(\rho^{\epsilon}\right)-p(\rho)^{\epsilon}\right) \cdot \varphi u^{\epsilon} d x d t\right|
$$




$$
\begin{aligned}
& \leqslant \int_{0}^{T} \int_{\mathbb{T}^{d}}\left|\varphi\left(p\left(\rho^{\epsilon}\right)-p(\rho)^{\epsilon}\right) \operatorname{div} u^{\epsilon}\right| d x d t+\int_{0}^{T} \int_{\mathbb{T}^{d}}\left|\left(p\left(\rho^{\epsilon}\right)-p(\rho)^{\epsilon}\right) u^{\epsilon} \cdot \nabla \varphi\right| d x d t \\
& \leqslant C\|\varphi\|_{C^{0}} \epsilon^{2 \gamma} \epsilon^{\alpha-1}\|\rho\|_{B_{3}^{\gamma, \infty}}^{2}\|u\|_{B_{3}^{\alpha, \infty}}+C\|\varphi\|_{C^{1}} \epsilon^{2 \gamma}\|\rho\|_{B_{3}^{\gamma, \infty}}^{2}\|u\|_{B_{3}^{\alpha, \infty}} \rightarrow 0 .
\end{aligned}
$$

最后估计 $\int_{0}^{T} \int_{\mathbb{T}^{d}} \varphi P^{\prime}\left(\rho^{\epsilon}\right) \operatorname{div}\left(\rho^{\epsilon} u^{\epsilon}-(\rho u)^{\epsilon}\right) d x d t$. 利用 (3.11) 将其分成两部分进行估计. 对于第 1 部分, 当 $\epsilon \rightarrow 0$ 时,

$$
\begin{aligned}
& \left|\int_{0}^{T} \int_{\mathbb{T}^{d}} \varphi P^{\prime}\left(\rho^{\epsilon}\right) \operatorname{div}\left[\left(\rho^{\epsilon}-\rho\right)\left(u^{\epsilon}-u\right)\right] d x d t\right| \\
& \quad \leqslant \int_{0}^{T} \int_{\mathbb{T}^{d}}\left|\nabla \varphi\left(\rho^{\epsilon}-\rho\right)\left(u^{\epsilon}-u\right) P^{\prime}\left(\rho^{\epsilon}\right)\right| d x d t+\int_{0}^{T} \int_{\mathbb{T}^{d}}\left|\varphi\left(\rho^{\epsilon}-\rho\right)\left(u^{\epsilon}-u\right) \cdot P^{\prime \prime}\left(\rho^{\epsilon}\right) \nabla \rho^{\epsilon}\right| d x d t \\
& \quad \leqslant C\|\varphi\|_{C^{1}} \epsilon^{\gamma} \epsilon^{\alpha}\|\rho\|_{B_{3}^{\gamma, \infty}}\|u\|_{B_{3}^{\alpha, \infty}}+C\|\varphi\|_{C^{0}} \epsilon^{\gamma} \epsilon^{\alpha} \epsilon^{\gamma-1}\|\rho\|_{B_{3}^{\gamma, \infty}}^{2}\|u\|_{B_{3}^{\alpha, \infty}} \rightarrow 0 .
\end{aligned}
$$

第 2 部分的证明与之类似. 至此完成了定理 1.1 的证明.

\section{2 定理 1.2 的证明}

首先将系统 (1.1) 关于时间和空间正则化. 不同于定理 1.1 证明过程中所采用的正则化, 这里对时 间和空间分别取不同的参数. 具体地, 对于空间, 我们利用如下关于空间的卷积:

$$
w^{\epsilon}(t, x)=\int_{\mathbb{T}^{d}} \eta^{\epsilon}(y) w(t, x-y) d y,
$$

这里 $\eta^{\epsilon}$ 由 (2.2) 给出. 对于时间的正则化, 利用文献 [20] 中的方法关于时间作卷积, 得

$$
w^{h}(t, x)=\int_{0}^{T} \chi_{h}(t-s) w(s, x) d s,
$$

这里 $\chi_{h}=\frac{1}{h} 1_{[-h, 0]}$. 若 $w \in L_{\mathrm{loc}}^{1}(0, T ; X)$ 且 $t$ 和 $t+h$ 是函数 $w$ 的 Lebesgue 点, 则有

$$
\partial_{t} w^{h}=\frac{w(t+h)-w(t)}{h} \in X .
$$

特别地, 函数 $w^{h}$ 在 $[h, T-h]$ 上是绝对连续的, 且对于几乎处处的 $t \in(h, T-h)$ 其导数满足 (3.16).

记

$$
w^{\epsilon, h}=\left(w^{\epsilon}\right)^{h}
$$

对 $(1.1)_{2}$ 进行正则化, 得

$$
\partial_{t} \rho^{\epsilon}+\operatorname{div}(\rho u)^{\epsilon}=0, \quad \partial_{t} \rho^{\epsilon, h}+\operatorname{div}(\rho u)^{\epsilon, h}=0,
$$

其中第 2 个等式在 $t \in(h, T-h)$ 内成立.

同样, 我们有

$$
\begin{aligned}
& \partial_{t}(\rho u)^{\epsilon}+\operatorname{div}\left((\rho u)^{\epsilon} \otimes u^{\epsilon}\right)+\nabla p(\rho)^{\epsilon} \\
& \quad=\operatorname{div}\left((\rho u)^{\epsilon} \otimes u^{\epsilon}\right)-\operatorname{div}(\rho u \otimes u)^{\epsilon}+(\operatorname{curl} B \times B)^{\epsilon}
\end{aligned}
$$

以及

$$
\partial_{t}\left(\rho^{\epsilon} u^{\epsilon}\right)^{h}+\operatorname{div}\left((\rho u)^{\epsilon} \otimes u^{\epsilon}\right)^{h}+\nabla(p(\rho))^{\epsilon, h}
$$




$$
=\left(\operatorname{div}\left((\rho u)^{\epsilon} \otimes u^{\epsilon}\right)-\operatorname{div}(\rho u \otimes u)^{\epsilon}\right)^{h}+\partial_{t}\left(\rho^{\epsilon} u^{\epsilon}-(\rho u)^{\epsilon}\right)^{h}+(\operatorname{curl} B \times B)^{\epsilon, h} .
$$

将 (3.19) 两边乘以 $\varphi u^{\epsilon, h}$, 这里 $\varphi \in C_{c}^{\infty}\left((h, T-h) \times \mathbb{T}^{d}\right)$, 并在两边加上关于磁场 $B$ 的项 $\left((u \times B)^{\epsilon}\right.$ $\left.\cdot \operatorname{curl} B^{\epsilon}\right)^{h} \varphi$, 再将所得方程在 $(0, T) \times \mathbb{T}^{d}$ 上积分, 得

$$
\begin{aligned}
\int_{0}^{T} \int_{\mathbb{T}^{d}} \partial_{t}\left(\rho^{\epsilon} u^{\epsilon}\right)^{h} \cdot u^{\epsilon, h} \varphi d x d t+\int_{0}^{T} \int_{\mathbb{T}^{d}} \operatorname{div}\left((\rho u)^{\epsilon} \otimes u^{\epsilon}\right)^{h} \cdot u^{\epsilon, h} \varphi d x d t \\
+\int_{0}^{T} \int_{\mathbb{T}^{d}} \nabla(p(\rho))^{\epsilon, h} \cdot u^{\epsilon, h} \varphi d x d t+\int_{0}^{T} \int_{\mathbb{T}^{d}}\left((u \times B)^{\epsilon} \cdot \operatorname{curl} B^{\epsilon}\right)^{h} \varphi d x d t \\
=\int_{0}^{T} \int_{\mathbb{T}^{d}} \operatorname{div}\left[\left((\rho u)^{\epsilon} \otimes u^{\epsilon}\right)-(\rho u \otimes u)^{\epsilon}\right]^{h} \cdot u^{\epsilon, h} \varphi d x d t \\
\quad+\int_{0}^{T} \int_{\mathbb{T}^{d}} \partial_{t}\left(\rho^{\epsilon} u^{\epsilon}-(\rho u)^{\epsilon}\right)^{h} \cdot u^{\epsilon, h} \varphi d x d t \\
\quad+\int_{0}^{T} \int_{\mathbb{T}^{d}}\left[(\operatorname{curl} B \times B)^{\epsilon}-\left(\operatorname{curl} B^{\epsilon} \times B^{\epsilon}\right)\right]^{h} \cdot u^{\epsilon, h} \varphi d x d t \\
\quad+\int_{0}^{T} \int_{\mathbb{T}^{d}}\left[(u \times B)^{\epsilon} \cdot \operatorname{curl} B^{\epsilon}-\left(u^{\epsilon} \times B^{\epsilon}\right) \cdot \operatorname{curl} B^{\epsilon}\right]^{h} \varphi d x d t \\
\quad+\int_{0}^{T} \int_{\mathbb{T}^{d}}\left[\left(\operatorname{curl} B^{\epsilon} \times B^{\epsilon}\right)^{h} u^{\epsilon, h}+\left(\left(u^{\epsilon} \times B^{\epsilon}\right) \cdot \operatorname{curl} B^{\epsilon}\right)^{h}\right] \varphi d x d t .
\end{aligned}
$$

首先处理 (3.20) 左端的第 1 项. 基于 (3.16), 有

$$
\begin{aligned}
\int_{0}^{T} & \int_{\mathbb{T}^{d}} \partial_{t}\left(\rho^{\epsilon} u^{\epsilon}\right)^{h} \cdot u^{\epsilon, h} \varphi d x d t \\
= & \int_{\mathbb{R}} \int_{\mathbb{T}^{d}} \frac{\rho^{\epsilon}(t+h) u^{\epsilon}(t+h)-\rho^{\epsilon}(t) u^{\epsilon}(t)}{h} \cdot u^{\epsilon, h} \varphi d x d t \\
& -\int_{\mathbb{R}} \int_{\mathbb{T}^{d}} \rho^{\epsilon} \frac{u^{\epsilon}(t+h)-u^{\epsilon}(t)}{h} \cdot u^{\epsilon, h} \varphi d x d t+\frac{1}{2} \int_{\mathbb{R}} \int_{\mathbb{T}^{d}} \rho^{\epsilon} \partial_{t}\left(\left|u^{\epsilon, h}\right|^{2}\right) \varphi d x d t \\
= & \int_{\mathbb{R}} \int_{\mathbb{T}^{d}} \frac{\rho^{\epsilon}(t+h)-\rho^{\epsilon}(t)}{h} u^{\epsilon}(t+h) \cdot u^{\epsilon, h} \varphi d x d t+\frac{1}{2} \int_{\mathbb{R}} \int_{\mathbb{T}^{d}} \rho^{\epsilon} \partial_{t}\left(\left|u^{\epsilon, h}\right|^{2}\right) \varphi d x d t \\
= & \int_{\mathbb{R}} \int_{\mathbb{T}^{d}} \partial_{t} \rho^{\epsilon, h} u^{\epsilon}(t+h) \cdot u^{\epsilon, h} \varphi d x d t+\frac{1}{2} \int_{\mathbb{R}} \int_{\mathbb{T}^{d}} \rho^{\epsilon} \partial_{t}\left(\left|u^{\epsilon, h}\right|^{2}\right) \varphi d x d t .
\end{aligned}
$$

再由正则化的连续性方程 (3.17), 得

$$
\begin{aligned}
\int_{0}^{T} & \int_{\mathbb{T}^{d}} \partial_{t}\left(\rho^{\epsilon} u^{\epsilon}\right)^{h} \cdot u^{\epsilon, h} \varphi d x d t \\
& =-\int_{\mathbb{R}} \int_{\mathbb{T}^{d}} \operatorname{div}(\rho u)^{\epsilon, h} u^{\epsilon}(t+h) \cdot u^{\epsilon, h} \varphi d x d t+\frac{1}{2} \int_{\mathbb{R}} \int_{\mathbb{T}^{d}} \rho^{\epsilon} \partial_{t}\left(\left|u^{\epsilon, h}\right|^{2}\right) \varphi d x d t \\
& =\int_{\mathbb{R}} \int_{\mathbb{T}^{d}}(\rho u)^{\epsilon, h} \cdot \nabla\left(u^{\epsilon}(t+h) \cdot u^{\epsilon, h} \varphi\right) d x d t+\frac{1}{2} \int_{\mathbb{R}} \int_{\mathbb{T}^{d}} \rho^{\epsilon} \partial_{t}\left(\left|u^{\epsilon, h}\right|^{2}\right) \varphi d x d t \\
& =\int_{\mathbb{R}} \int_{\mathbb{T}^{d}}(\rho u)^{\epsilon, h} \cdot \nabla\left(u^{\epsilon}(t+h) \cdot u^{\epsilon, h} \varphi\right) d x d t-\frac{1}{2} \int_{\mathbb{R}} \int_{\mathbb{T}^{d}}\left[(\rho u)^{\epsilon} \cdot \nabla\left(\varphi\left|u^{\epsilon, h}\right|^{2}\right)+\rho^{\epsilon}\left|u^{\epsilon, h}\right|^{2} \partial_{t} \varphi\right] d x d t
\end{aligned}
$$

对于 (3.20) 左边第 2 项, 有

$$
\int_{0}^{T} \int_{\mathbb{T}^{d}} \operatorname{div}\left((\rho u)^{\epsilon} \otimes u^{\epsilon}\right)^{h} \cdot u^{\epsilon, h} \varphi d x d t=-\int_{\mathbb{R}} \int_{\mathbb{T}^{d}}\left((\rho u)^{\epsilon} \otimes u^{\epsilon}\right)^{h}: \nabla\left(u^{\epsilon, h} \varphi\right) d x d t .
$$


对于 (3.20) 左边第 4 项, 借助于公式 $\operatorname{div}(a \times b)=\operatorname{curl} a \cdot b-a \cdot \operatorname{curl} b$ 及 (3.6), 得

$$
\begin{aligned}
\int_{0}^{T} & \int_{\mathbb{T}^{d}}\left[(u \times B)^{\epsilon} \cdot \operatorname{curl} B^{\epsilon}\right]^{h} \varphi d x d t \\
& =\int_{\mathbb{R}} \int_{\mathbb{T}^{d}}\left[\operatorname{curl}(u \times B)^{\epsilon} \cdot B^{\epsilon}\right]^{h} \varphi d x d t-\int_{\mathbb{R}} \int_{\mathbb{T}^{d}} \operatorname{div}\left((u \times B)^{\epsilon} \times B^{\epsilon}\right)^{h} \varphi d x d t \\
& =\int_{\mathbb{R}} \int_{\mathbb{T}^{d}}\left(\frac{1}{2} \partial_{t}\left|B^{\epsilon}\right|^{2}\right)^{h} \varphi d x d t-\int_{\mathbb{R}^{d}} \int_{\mathbb{T}^{d}} \operatorname{div}\left((u \times B)^{\epsilon} \times B^{\epsilon}\right)^{h} \varphi d x d t \\
& =-\int_{\mathbb{R}} \int_{\mathbb{T}^{d}} \partial_{t} \varphi\left(\frac{1}{2}\left|B^{\epsilon}\right|^{2}\right)^{h} d x d t+\int_{\mathbb{R}} \int_{\mathbb{T}^{d}}\left((u \times B)^{\epsilon} \times B^{\epsilon}\right)^{h} \cdot \nabla \varphi d x d t .
\end{aligned}
$$

下面考虑 (3.20) 左边第 3 项. 根据定理 1.2 中的假设 (1.3), 可将压力 $p(\rho)$ 表示如下: 对任意 $\delta>0$, 有

$$
\begin{aligned}
& p(\rho)=p_{\delta}(\rho)+\left(p(\rho)-p_{\delta}(\rho)\right), \quad p_{\delta} \in C^{2}[0, \infty), \\
& p_{\delta}(0)=p_{\delta}^{\prime}(0)=0, \quad\left|p-p_{\delta}\right|<\delta .
\end{aligned}
$$

相应地, 有

$$
\begin{aligned}
\int_{0}^{t} & \int_{\mathbb{T}^{d}}(\nabla p(\rho))^{\epsilon, h} \cdot u^{\epsilon, h} \varphi d x d t \\
= & -\int_{\mathbb{R}} \int_{\mathbb{T}^{d}}(p(\rho))^{\epsilon, h} \operatorname{div} u^{\epsilon, h} \varphi d x d t-\int_{\mathbb{R}} \int_{\mathbb{T}^{d}}(p(\rho))^{\epsilon, h} u^{\epsilon, h} \cdot \nabla \varphi d x d t \\
= & -\int_{\mathbb{R}} \int_{\mathbb{T}^{d}}\left(p_{\delta}(\rho)\right)^{\epsilon, h} \operatorname{div} u^{\epsilon, h} \varphi d x d t-\int_{\mathbb{R}} \int_{\mathbb{T}^{d}}\left(p_{\delta}(\rho)\right)^{\epsilon, h} u^{\epsilon, h} \cdot \nabla \varphi d x d t+\mathcal{O}(\delta) \\
= & -\int_{\mathbb{R}} \int_{\mathbb{T}^{d}} p_{\delta}\left(\rho^{\epsilon, h}\right) \operatorname{div} u^{\epsilon, h} \varphi d x d t-\int_{\mathbb{R}} \int_{\mathbb{T}^{d}}\left(p_{\delta}(\rho)\right)^{\epsilon, h} u^{\epsilon, h} \cdot \nabla \varphi d x d t \\
& +\int_{\mathbb{R}} \int_{\mathbb{T}^{d}}\left(p_{\delta}\left(\rho^{\epsilon, h}\right)-\left(p_{\delta}(\rho)\right)^{\epsilon, h}\right) \operatorname{div} u^{\epsilon, h} \varphi d x d t+\mathcal{O}(\delta) .
\end{aligned}
$$

为了计算上式右端第 1 项, 将连续性方程 $(1.1)_{2}$ 关于时间和空间分别磨光后成为

$$
\partial_{t} \rho^{\epsilon, h}+\operatorname{div}\left(\rho^{\epsilon, h} u^{\epsilon, h}\right)=-\operatorname{div}(\rho u)^{\epsilon, h}+\operatorname{div}\left(\rho^{\epsilon, h} u^{\epsilon, h}\right),
$$

因此, 类似于 (3.8), 可得

$$
\begin{gathered}
\partial_{t} P_{\delta}\left(\rho^{\epsilon, h}\right)+\operatorname{div}\left(P_{\delta}\left(\rho^{\epsilon, h}\right) u^{\epsilon, h}\right)+p_{\delta}\left(\rho^{\epsilon, h}\right) \operatorname{div} u^{\epsilon, h} \\
=-P_{\delta}^{\prime}\left(\rho^{\epsilon, h}\right)\left[\operatorname{div}(\rho u)^{\epsilon, h}-\operatorname{div}\left(\rho^{\epsilon, h} u^{\epsilon, h}\right)\right],
\end{gathered}
$$

这里

$$
P_{\delta}(\rho)=\rho \int_{1}^{\rho} \frac{p_{\delta}(z)}{z^{2}} d z .
$$

由 (3.24), 有 $P_{\delta} \in C^{2}[0, \infty)$. 回到 (3.25), 可推导出

$$
\begin{aligned}
& \int_{0}^{t} \int_{\mathbb{T}^{d}}(\nabla p(\rho))^{\epsilon, h} \cdot u^{\epsilon, h} \varphi d x d t \\
& =\int_{\mathbb{R}} \int_{\mathbb{T}^{d}}\left[\partial_{t} P_{\delta}\left(\rho^{\epsilon, h}\right)+\operatorname{div}\left(P_{\delta}\left(\rho^{\epsilon, h}\right) u^{\epsilon, h}\right)\right] \varphi d x d t-\int_{\mathbb{R}} \int_{\mathbb{T}^{d}}\left(p_{\delta}(\rho)\right)^{\epsilon, h} u^{\epsilon, h} \cdot \nabla \varphi d x d t \\
& \quad+\int_{\mathbb{R}} \int_{\mathbb{T}^{d}}\left(p_{\delta}\left(\rho^{\epsilon, h}\right)-\left(p_{\delta}(\rho)\right)^{\epsilon, h}\right) \operatorname{div} u^{\epsilon, h} \varphi d x d t
\end{aligned}
$$




$$
\begin{aligned}
& +\int_{\mathbb{R}} \int_{\mathbb{T}^{d}} P_{\delta}^{\prime}\left(\rho^{\epsilon, h}\right)\left[\operatorname{div}(\rho u)^{\epsilon, h}-\operatorname{div}\left(\rho^{\epsilon, h} u^{\epsilon, h}\right)\right] \varphi d x d t+\mathcal{O}(\delta) \\
= & -\int_{\mathbb{R}} \int_{\mathbb{T}^{d}} P\left(\rho^{\epsilon, h}\right) \partial_{t} \varphi d x d t-\int_{\mathbb{R}} \int_{\mathbb{T}^{d}} P\left(\rho^{\epsilon, h}\right) u^{\epsilon, h} \cdot \nabla \varphi d x d t \\
& -\int_{\mathbb{R}} \int_{\mathbb{T}^{d}}(p(\rho))^{\epsilon, h} u^{\epsilon, h} \cdot \nabla \varphi d x d t+\int_{\mathbb{R}} \int_{\mathbb{T}^{d}}\left(p_{\delta}\left(\rho^{\epsilon, h}\right)-\left(p_{\delta}(\rho)\right)^{\epsilon, h}\right) \operatorname{div} u^{\epsilon, h} \varphi d x d t \\
& +\int_{\mathbb{R}} \int_{\mathbb{T}^{d}} P_{\delta}^{\prime}\left(\rho^{\epsilon, h}\right)\left[\operatorname{div}(\rho u)^{\epsilon, h}-\operatorname{div}\left(\rho^{\epsilon, h} u^{\epsilon, h}\right)\right] \varphi d x d t+\mathcal{O}(\delta) .
\end{aligned}
$$

将 (3.21)-(3.23) 和 (3.27) 代入 (3.20), 得

$$
\begin{aligned}
& \int_{\mathbb{R}} \int_{\mathbb{T}^{d}}(\rho u)^{\epsilon, h} \cdot \nabla\left(u^{\epsilon}(t+h) \cdot u^{\epsilon, h} \varphi\right) d x d t-\frac{1}{2} \int_{\mathbb{R}} \int_{\mathbb{T}^{d}}\left[(\rho u)^{\epsilon} \cdot \nabla\left(\varphi\left|u^{\epsilon, h}\right|^{2}\right)+\rho^{\epsilon}\left|u^{\epsilon, h}\right|^{2} \partial_{t} \varphi\right] d x d t \\
& \quad-\int_{\mathbb{R}} \int_{\mathbb{T}^{d}}\left((\rho u)^{\epsilon} \otimes u^{\epsilon}\right)^{h}: \nabla\left(u^{\epsilon, h} \varphi\right) d x d t-\int_{\mathbb{R}} \int_{\mathbb{T}^{d}} P\left(\rho^{\epsilon, h}\right) \partial_{t} \varphi d x d t-\int_{\mathbb{R}} \int_{\mathbb{T}^{d}} P\left(\rho^{\epsilon, h}\right) u^{\epsilon, h} \cdot \nabla \varphi d x d t \\
& -\int_{\mathbb{R}} \int_{\mathbb{T}^{d}}(p(\rho))^{\epsilon, h} u^{\epsilon, h} \cdot \nabla \varphi d x d t+\int_{\mathbb{R}} \int_{\mathbb{T}^{d}}\left(p_{\delta}\left(\rho^{\epsilon, h}\right)-\left(p_{\delta}(\rho)\right)^{\epsilon, h}\right) \operatorname{div} u^{\epsilon, h} \varphi d x d t \\
& +\int_{\mathbb{R}} \int_{\mathbb{T}^{d}} P_{\delta}^{\prime}\left(\rho^{\epsilon, h}\right)\left[\operatorname{div}(\rho u)^{\epsilon, h}-\operatorname{div}\left(\rho^{\epsilon, h} u^{\epsilon, h}\right)\right] \varphi d x d t \\
& -\int_{\mathbb{R}} \int_{\mathbb{T}^{d}} \frac{1}{2}\left(\left|B^{\epsilon}\right|^{2}\right)^{h} \partial_{t} \varphi d x d t+\int_{\mathbb{R}} \int_{\mathbb{T}^{d}}\left((u \times B)^{\epsilon} \times B^{\epsilon}\right)^{h} \cdot \nabla \varphi d x d t \\
& -\int_{\mathbb{R}} \int_{\mathbb{T}^{d}} \operatorname{div}\left[\left((\rho u)^{\epsilon} \otimes u^{\epsilon}\right)-(\rho u \otimes u)^{\epsilon}\right]^{h} \cdot u^{\epsilon, h} \varphi d x d t-\int_{\mathbb{R}} \int_{\mathbb{T}^{d}} \partial_{t}\left(\rho^{\epsilon} u^{\epsilon}-(\rho u)^{\epsilon}\right)^{h} \cdot u^{\epsilon, h} \varphi d x d t \\
& -\int_{\mathbb{R}} \int_{\mathbb{T}^{d}}\left[(\operatorname{curl} B \times B)^{\epsilon}-\left(\operatorname{curl} B^{\epsilon} \times B^{\epsilon}\right)\right]^{h} \cdot u^{\epsilon, h} \varphi d x d t \\
& -\int_{\mathbb{R}} \int_{\mathbb{T}^{d}}\left[(u \times B)^{\epsilon} \cdot \operatorname{curl} B^{\epsilon}-\left(u^{\epsilon} \times B^{\epsilon}\right) \cdot \operatorname{curl} B^{\epsilon}\right]^{h} \varphi d x d t \\
& -\int_{\mathbb{R}} \int_{\mathbb{T}^{d}}\left[\left(\operatorname{curl} B^{\epsilon} \times B^{\epsilon}\right)^{h} u^{\epsilon, h}+\left(\left(u^{\epsilon} \times B^{\epsilon}\right) \cdot \operatorname{curl} B^{\epsilon}\right)^{h}\right] \varphi d x d t \\
& \quad=\mathcal{O}(\delta) .
\end{aligned}
$$

将上式进一步变形, 有

$$
\begin{aligned}
& \int_{\mathbb{R}} \int_{\mathbb{T}^{d}}\left(\frac{1}{2} \rho^{\epsilon}\left|u^{\epsilon, h}\right|^{2}+P\left(\rho^{\epsilon, h}\right)+\frac{1}{2}\left(\left|B^{\epsilon}\right|^{2}\right)^{h}\right) \partial_{t} \varphi d x d t \\
& \quad+\int_{\mathbb{R}} \int_{\mathbb{T}^{d}}\left(\left((\rho u)^{\epsilon} \otimes u^{\epsilon}\right)^{h} \cdot u^{\epsilon, h}+\frac{1}{2}(\rho u)^{\epsilon}\left|u^{\epsilon, h}\right|^{2}-(\rho u)^{\epsilon, h}\left(u^{\epsilon}(t+h) \cdot u^{\epsilon, h}\right)\right) \cdot \nabla \varphi d x d t \\
& \quad+\int_{\mathbb{R}} \int_{\mathbb{T}^{d}}\left(P\left(\rho^{\epsilon, h}\right)+(p(\rho))^{\epsilon, h}\right) u^{\epsilon, h} \cdot \nabla \varphi d x d t-\int_{\mathbb{R}} \int_{\mathbb{T}^{d}}\left((u \times B)^{\epsilon} \times B^{\epsilon}\right)^{h} \cdot \nabla \varphi d x d t \\
& \quad=: \sum_{i=1}^{8} H_{\epsilon, h}^{i}+\mathcal{O}(\delta),
\end{aligned}
$$

其中,

$$
\begin{aligned}
H_{\epsilon, h}^{1}= & \int_{\mathbb{R}} \int_{\mathbb{T}^{d}}(\rho u)^{\epsilon, h} \cdot \nabla\left(u^{\epsilon}(t+h) \cdot u^{\epsilon, h}\right) \varphi d x d t-\frac{1}{2} \int_{\mathbb{R}} \int_{\mathbb{T}^{d}}(\rho u)^{\epsilon} \cdot \nabla\left(\left|u^{\epsilon, h}\right|^{2}\right) \varphi d x d t \\
& -\int_{\mathbb{R}} \int_{\mathbb{T}^{d}}\left((\rho u)^{\epsilon} \otimes u^{\epsilon}\right)^{h}: \nabla u^{\epsilon, h} \varphi d x d t
\end{aligned}
$$




$$
\begin{aligned}
& H_{\epsilon, h}^{2}=\int_{\mathbb{R}} \int_{\mathbb{T}^{d}}\left(p_{\delta}\left(\rho^{\epsilon, h}\right)-\left(p_{\delta}(\rho)\right)^{\epsilon, h}\right) \operatorname{div} u^{\epsilon, h} \varphi d x d t, \\
& H_{\epsilon, h}^{3}=\int_{\mathbb{R}} \int_{\mathbb{T}^{d}} P_{\delta}^{\prime}\left(\rho^{\epsilon, h}\right)\left[\operatorname{div}(\rho u)^{\epsilon, h}-\operatorname{div}\left(\rho^{\epsilon, h} u^{\epsilon, h}\right)\right] \varphi d x d t, \\
& H_{\epsilon, h}^{4}=-\int_{\mathbb{R}} \int_{\mathbb{T}^{d}} \operatorname{div}\left[\left((\rho u)^{\epsilon} \otimes u^{\epsilon}\right)-(\rho u \otimes u)^{\epsilon}\right]^{h} \cdot u^{\epsilon, h} \varphi d x d t, \\
& H_{\epsilon, h}^{5}=-\int_{\mathbb{R}} \int_{\mathbb{T}^{d}} \partial_{t}\left(\rho^{\epsilon} u^{\epsilon}-(\rho u)^{\epsilon}\right)^{h} \cdot u^{\epsilon, h} \varphi d x d t, \\
& H_{\epsilon, h}^{6}=-\int_{\mathbb{R}} \int_{\mathbb{T}^{d}}\left[(\operatorname{curl} B \times B)^{\epsilon}-\left(\operatorname{curl} B^{\epsilon} \times B^{\epsilon}\right)\right]^{h} \cdot u^{\epsilon, h} \varphi d x d t, \\
& H_{\epsilon, h}^{7}=-\int_{\mathbb{R}} \int_{\mathbb{T}^{d}}\left[(u \times B)^{\epsilon} \cdot \operatorname{curl} B^{\epsilon}-\left(u^{\epsilon} \times B^{\epsilon}\right) \cdot \operatorname{curl} B^{\epsilon}\right]^{h} \varphi d x d t, \\
& H_{\epsilon, h}^{8}=-\int_{\mathbb{R}} \int_{\mathbb{T}^{d}}\left[\left(\operatorname{curl} B^{\epsilon} \times B^{\epsilon}\right)^{h} u^{\epsilon, h}+\left(\left(u^{\epsilon} \times B^{\epsilon}\right) \cdot \operatorname{curl} B^{\epsilon}\right)^{h}\right] \varphi d x d t .
\end{aligned}
$$

当 $\epsilon, h \rightarrow 0$ 时, 根据磨光性质, 可得 (3.28) 左端的极限为

$$
\begin{aligned}
& \int_{\mathbb{R}} \int_{\mathbb{T}^{d}} \partial_{t} \varphi\left(\frac{1}{2} \rho|u|^{2}+\frac{1}{2}|B|^{2}+P(\rho)\right) d x d t \\
& \quad+\int_{\mathbb{R}} \int_{\mathbb{T}^{d}} \nabla \varphi \cdot\left(\frac{1}{2} \rho|u|^{2} u+p(\rho) u+P(\rho) u-(u \times B) \times B\right) d x d t .
\end{aligned}
$$

为证明定理 1.2 , 需证明当 $\epsilon, h \rightarrow 0$ 时, $H_{\epsilon, h}^{i}(i=1,2, \ldots, 8)$ 均趋于 0 . 这里首先考虑 $\epsilon \rightarrow 0$ 时 $H_{\epsilon, h}^{i}(i=1,2, \ldots, 8)$ 的极限, 再考虑 $h \rightarrow 0$ 时的极限. 对于 $H_{\epsilon, h}^{1}$, 可将其改写为

$$
\begin{aligned}
H_{\epsilon, h}^{1}= & \int_{\mathbb{R}} \int_{\mathbb{T}^{d}}(\rho u)^{\epsilon, h}(t-h) \cdot \nabla u^{\epsilon} \cdot u^{\epsilon, h}(t-h) \varphi(t-h) d x d t+\int_{\mathbb{R}} \int_{\mathbb{T}^{d}}(\rho u)^{\epsilon, h} \cdot u^{\epsilon}(t+h) \cdot \nabla u^{\epsilon, h} \varphi d x d t \\
& -\int_{\mathbb{R}} \int_{\mathbb{T}^{d}}(\rho u)^{\epsilon} \cdot \nabla u^{\epsilon, h} \cdot u^{\epsilon, h} \varphi d x d t-\int_{\mathbb{R}} \int_{\mathbb{T}^{d}}\left((\rho u)^{\epsilon} \otimes u^{\epsilon}\right)^{h}: \nabla u^{\epsilon, h} \varphi d x d t .
\end{aligned}
$$

由定理 1.2 的假设条件

$$
\text { ess } \sup _{t \in[0, T]}\left(\|\rho(t, \cdot)\|_{B V \cap C\left(\mathbb{T}^{d}\right)}+\|u(t, \cdot)\|_{B V \cap C\left(\mathbb{T}^{d} ; \mathbb{R}^{d}\right)}+\|B(t, \cdot)\|_{B V \cap C\left(\mathbb{T}^{d} ; \mathbb{R}^{d}\right)}\right)<\infty
$$

可知, 当 $h$ 固定而 $\epsilon \rightarrow 0$ 时, 对任意 $1 \leqslant p<\infty$, 有

$$
\begin{aligned}
& (\rho u)^{\epsilon, h} \rightarrow(\rho u)^{h}, \quad \text { 在 } C\left([h, T-h] \times \mathbb{T}^{d} ; \mathbb{R}^{d}\right) \text { 中, } \\
& u^{\epsilon, h} \rightarrow u^{h}, \quad \text { 在 } C\left([h, T-h] \times \mathbb{T}^{d} ; \mathbb{R}^{d}\right) \text { 中, } \\
& \nabla u^{\epsilon} \rightarrow \nabla u, \quad \text { 在 } L_{\text {weak }^{*}}\left(0, T ; \mathcal{M}\left(\mathbb{T}^{d} ; \mathbb{R}^{d \times d}\right)\right) \text { 中, } \\
& \nabla u^{\epsilon, h} \rightarrow \nabla u^{h}, \quad \text { 在 } C_{\text {weak }^{*}}\left([h, T-h] ; \mathcal{M}\left(\mathbb{T}^{d} ; \mathbb{R}^{d \times d}\right)\right) \text { 中, } \\
& (\rho u)^{\epsilon} \rightarrow \rho u, \text { 在 } L^{p}\left(0, T ; C\left(\mathbb{T}^{d} ; \mathbb{R}^{d}\right)\right) \text { 中, } \\
& u^{\epsilon} \rightarrow u, \text { 在 } L^{p}\left(0, T ; C\left(\mathbb{T}^{d} ; \mathbb{R}^{d}\right)\right) \text { 中. }
\end{aligned}
$$

因此, 当 $\epsilon \rightarrow 0$ 时可推导出

$$
H_{\epsilon, h}^{1} \rightarrow H_{h}^{1}=\int_{\mathbb{R}} \int_{\mathbb{T}^{d}}(\rho u)^{h}(t-h) \cdot \nabla u \cdot u^{h}(t-h) \varphi(t-h) d x d t+\int_{\mathbb{R}} \int_{\mathbb{T}^{d}}(\rho u)^{h} \cdot u(t+h) \cdot \nabla u^{h} \varphi d x d t
$$




$$
-\int_{\mathbb{R}} \int_{\mathbb{T}^{d}}(\rho u) \cdot \nabla u^{h} \cdot u^{h} \varphi d x d t-\int_{\mathbb{R}} \int_{\mathbb{T}^{d}}((\rho u) \otimes u)^{h}: \nabla u^{h} \varphi d x d t .
$$

注意到, 若 $X$ 是一个 Banach 空间, 则对任意 $v \in L^{\infty}(0, T ; X)$ 和任意 $1 \leqslant p<\infty$, 当 $h \rightarrow 0$ 时, 有

$$
v^{h} \rightarrow v \quad \text { 和 } \quad v(\cdot \pm h) \rightarrow v, \quad \text { 在 } L^{p}(0, T ; X) \text { 中. }
$$

由此得到 $H_{h}^{1} \rightarrow 0$.

类似地, 可以处理 $H_{\epsilon, h}^{2} 、 H_{\epsilon, h}^{4} 、 H_{\epsilon, h}^{6}$ 和 $H_{\epsilon, h}^{7}$. 对于 $H_{\epsilon, h}^{3}$, 由分部积分公式, 得

$$
H_{\epsilon, h}^{3}=-\int_{\mathbb{R}} \int_{\mathbb{T}^{d}} \nabla\left(P_{\delta}^{\prime}\left(\rho^{\epsilon, h}\right) \varphi\right)\left[(\rho u)^{\epsilon, h}-\rho^{\epsilon, h} u^{\epsilon, h}\right] d x d t,
$$

这里

$$
\nabla P_{\delta}^{\prime}\left(\rho^{\epsilon, h}\right)=P_{\delta}^{\prime \prime}\left(\rho^{\epsilon, h}\right) \nabla \rho^{\epsilon, h} .
$$

再经过上述相同的论证可得 $H_{\epsilon, h}^{3}$ 趋于 0 .

对于 $H_{\epsilon, h}^{5}$, 同样由分部积分公式可得

$$
H_{\epsilon, h}^{5}=\int_{\mathbb{R}} \int_{\mathbb{T}^{d}}\left(\left(\rho^{\epsilon} u^{\epsilon}-(\rho u)^{\epsilon}\right)^{h}\right) \cdot \partial_{t}\left(u^{\epsilon, h} \varphi\right) d x d t .
$$

对固定的 $h$, 由于 $u \in L^{\infty}$, 因此 $\partial_{t}\left(u^{\epsilon, h} \varphi\right)$ 一致有界. 另外, 同样由于 $\rho, u \in L^{\infty}$, 因此当 $\epsilon \rightarrow 0$ 时, 有

$$
\rho^{\epsilon} u^{\epsilon}-(\rho u)^{\epsilon} \rightarrow 0, \quad \text { 在 } L^{p}\left((0, T) \times \mathbb{T}^{d}\right)(1 \leqslant p<\infty) \text { 中, }
$$

从而 $H_{\epsilon, h}^{5}$ 趋于 0 .

最后处理 $H_{\epsilon, h}^{8}$. 由等式 $\left(u^{\epsilon} \times B^{\epsilon}\right) \cdot \operatorname{curl} B^{\epsilon}=-u^{\epsilon} \cdot\left(\operatorname{curl} B^{\epsilon} \times B^{\epsilon}\right)$ 可将 $H_{\epsilon, h}^{8}$ 变为

$$
\begin{aligned}
H_{\epsilon, h}^{8} & =-\int_{\mathbb{R}} \int_{\mathbb{T}^{d}}\left[\left(\operatorname{curl} B^{\epsilon} \times B^{\epsilon}\right)^{h} u^{\epsilon, h}+\left(\left(u^{\epsilon} \times B^{\epsilon}\right) \cdot \operatorname{curl} B^{\epsilon}\right)^{h}\right] \varphi d x d t \\
& =\int_{\mathbb{R}} \int_{\mathbb{T}^{d}}\left[\left(\left(\operatorname{curl} B^{\epsilon} \times B^{\epsilon}\right) u^{\epsilon}\right)^{h}-\left(\operatorname{curl} B^{\epsilon} \times B^{\epsilon}\right)^{h} u^{\epsilon, h}\right] \varphi d x d t .
\end{aligned}
$$

类似地, $H_{\epsilon, h}^{8}$ 趋于 0 . 最后, 令 (3.28) 中的 $\delta \rightarrow 0$. 至此定理 1.2 证毕.

致谢 作者衰心感谢审稿专家和编委提出的宝贵建议.

\section{参考文献}

1 Pedlosky J. Geophysical Fluid Dynamics. New York: Springer-Verlag, 1987

2 Landau L, Lifshitz E. Electrodynamics of Continuous Media, 2nd ed. New York: Pergamon, 1984

3 Sermange M, Temam R. Some mathematical questions related to the MHD equations. Comm Pure Appl Math, 1983, 36: $635-664$

4 Onsager L. Statistical hydrodynamics. Nuovo Cim, 1949, 6: 279-287

5 Eyink G L. Energy dissipation without viscosity in ideal hydrodynamics, I: Fourier analysis and local energy transfer. Phys D, 1994, 78: 222-240

6 Constantin P, E W N, Titi E S. Onsager's conjecture on the energy conservation for solutions of Euler's equation. Comm Math Phys, 1994, 165: 207-209

7 Duchon J, Robert R. Inertial energy dissipation for weak solutions of incompressible Euler and Navier-Stokes equations. Nonlinearity, 2000, 13: 249-255 
8 Cheskidov A, Constantin P, Friedlander S, et al. Energy conservation and Onsager's conjecture for the Euler equations. Nonlinearity, 2008, 21: 1233-1252

9 Bardos C, Titi E S. Onsager's conjecture for the incompressible Euler equations in bounded domains. Arch Ration Mech Anal, 2018, 228: 197-207

10 Bardos C, Titi E S, Wiedemann E. Onsager's conjecture with physical boundaries and an application to the vanishing viscosity limit. Comm Math Phys, 2019, 370: 291-310

11 Nguyen Q H, Nguyen P T. Onsager's conjecture on the energy conservation for solutions of Euler equations in bounded domains. J Nonlinear Sci, 2019, 29: 207-213

12 Akramov I, Dębiec T, Skipper J, et al. Energy conservation for the compressible Euler and Navier-Stokes equations with vacuum. Anal PDE, 2020, 13: 789-811

13 Feireisl E, Gwiazda P, Świerczewska-Gwiazda A, et al. Regularity and energy conservation for the compressible Euler equations. Arch Ration Mech Anal, 2017, 223: 1375-1395

$14 \mathrm{Yu}$ C. Energy conservation for the weak solutions of the compressible Navier-Stokes equations. Arch Ration Mech Anal, 2017, 225: 1073-1087

$15 \mathrm{Wu}$ Z E, Tan Z. Regularity and energy dissipation for the nonhomogeneous incompressible MHD equations (in Chinese). Sci Sin Math, 2019, 49: 1967-1978 [吴忠二, 谭忠. 非齐次不可压 MHD 方程的正则性与能量耗散. 中国科学: 数学, 2019, 49: 1967-1978]

16 Wang Y, Zuo B. Energy and cross-helicity conservation for the three-dimensional ideal MHD equations in a bounded domain. J Differential Equations, 2020, 268: 4079-4101

17 Gao Z, Tan Z, Wu G. Energy dissipation for weak solutions of incompressible MHD equations. Acta Math Sci Ser B Engl Ed, 2013, 33: 865-871

18 Guo S, Tan Z. Local 4/5-law and energy dissipation anomaly in turbulence of incompressible MHD equations. Z Angew Math Phys, 2016, 67: 147

$19 \mathrm{Wu}$ Z, Tan Z. Regularity and energy conservation for compressible isentropic magnetohydrodynamic equations. Math Methods Appl Sci, 2021, 44: 533-545

20 Frehse J, Málek J, Ružicka M. Large data existence result for unsteady flows of inhomogeneous shear-thickening heat-conducting incompressible fluids. Comm Partial Differential Equations, 2010, 35: 1891-1919

21 Chen R M, Yu C. Onsager's energy conservation for inhomogeneous Euler equations. J Math Pures Appl (9), 2019, 131: $1-16$

\section{Regularity and energy conservation for the compressible MHD equations}

Qunyi Bie, Lingping Kang, Qiru Wang \& Zheng-an Yao

Abstract In this paper, we are concerned with the regularity and energy conservation of the weak solutions for inhomogeneous compressible ideal MHD (magnetohydrodynamics) systems. We give two sufficient conditions on the regularity of solutions in order to ensure the energy to be conserved, where $(t, x) \in(0, T) \times \mathbb{T}^{d}(d \geqslant 1)$. The main tool we have employed relies on the commutator estimates similar to those used by Constantin et al. (1994) for the homogeneous incompressible Euler equations.

Keywords compressible MHD equations, regularity, energy conservation MSC(2020) 35D30, 35B65, 35Q35, 76W05

doi: 10.1360/SSM-2020-0339 